\title{
Sostenibilidad del Balneario El Rodadero, Santa Marta, Colombia*
}

Sustainability in the Swimming Area El Rodadero in Santa Marta, Colombia

Luz Mery Sánchez Sánchez ${ }^{a}$

Universidad Jorge Tadeo Lozano, Colombia

luzm.sanchezs@utadeo.edu.co

ORCID: http://orcid.org/0000-0002-9804-9560
DOI: https://doi.org/10.11144/Javeriana.ayd22-42.sber Redalyc: http://www.redalyc.org/articulo.oa? id $=151557418006$

Fecha de recepción: 28 Octubre 2017 Fecha de publicación: 28 Junio 2018

\section{Resumen:}

La sostenibilidad en las dimensiones ambiental, sociocultural y económica; la subsidiariedad, la equidad, la eficiencia en la prestación de los servicios públicos y en la promoción del desarrollo económico local; la transparencia, el compromiso cívico y la ciudadanía, en especial las mujeres y los pobres, al igual que la seguridad de las personas y su ambiente, en conjunto con la unión y asociación de los grupos de interés públicos y privados, en forma vertical y horizontal, se constituyen en los principios de gobernabilidad urbana básicos establecidos por la Organización de las Naciones Unidas (ONU) (2005). En el ámbito turístico, es la brújula que debe direccionar la conversión de un destino turístico a uno sostenible, complementado con los lineamientos dados en el Programa 21 de la ONU, los Criterios Globales de Turismo Sostenible, la Carta Mundial de Turismo Sostenible +20 y la Organización Mundial del Turismo (OMT). Desde este contexto teórico, se determinan los factores de riesgo que pueden afectar la sostenibilidad del balneario El Rodadero, a partir de la descripción de los indicadores establecidos en la Norma Técnica Sectorial de Turismo- Destinos de Playa -Requisitos de Sostenibilidad (NTS-TS-001-2). Los datos provienen de la observación directa, y de algunos resultados de las encuestas aplicadas a turistas, empresarios, residentes, e informes de investigaciones relacionadas y boletines técnicos.

Palabras clave: gobernabilidad urbana, turismo sostenible, norma técnica sectorial de turismo (NTS-TS-001-2).

\section{Abstract:}

Sustainability in the environmental, sociocultural and economic dimensions; subsidiarity, equity, effectiveness in the supply of public services and in the promotion of the local economic development; transparence, civic commitment and the citizens particularly women and the poor-, the security and the environment; all these topics developed under an approach that associates and joins together public and private stakeholders, both vertically and horizontally, constitutes the urban governance principles provided by the United Nations (UN, 2005). In the tourism industry, they must drive the conversion of a tourism place into a sustainable one and complemented with the guidelines provided in the UN Program 21 (1992), Global Criteria for Sustainable Tourism (Global Sustainable Tourism Council [GSTC], 2008), and the World Charter for Sustainable Tourism +20 (Global Sustainable Tourism Conference, 2015) (WTO, 2016). In this theoretical context, the risk factors affecting the sustainability in the swimming area El Rodadero are determined based on the description of indicators provided in the technical standard for tourism: Beach Destinations, Sustainability Requirements (NTS-TS-001-2). Data were collected through direct observation and some surveys applied to tourists, businesspeople and locals and also from related research reports and technical bulletins.

Keywords: urban governance, sustainable tourism, technical standard for tourism (NTS-TS-001-2).

\section{Introducción}

Santa Marta, ubicada en la Costa Norte del Caribe Colombiano, es denominada la Perla del Caribe y la bahía más hermosa de América, en razón de las ventajas comparativas de paisaje natural de playa y montaña, y de su clima agradable de brisa y baja salinidad. A esta ciudad pertenece el balneario El Rodadero, la playa más visitada y la primera vitrina turística del Departamento del Magdalena, con $2 \mathrm{~km}$ de extensión. Pese a estos aspectos positivos, según Arregocés (2009) y Martínez (2013), el desarrollo del sector ha traído consigo impactos ambientales, económicos y sociales, que muestran el deterioro y la falta de responsabilidad social, tanto de los habitantes del sector, como de los turistas que visitan este importante lugar de Colombia.

\footnotetext{
a Autora de correspondencia. Correo electrónico: luzm.sanchezs@utadeo.edu.co
} 
Asimismo, las estadísticas de turismo muestran bajos resultados de crecimiento, en comparación con cifras nacionales e internacionales, con incrementos del 4\% (Ministerio de Industria Comercio y Turismo y Departamento Nacional de Planeación, 2014). Esta breve descripción se constituye en la motivación sobre la cual se da inicio a la investigación titulada Hacia la construcción de la marca Rodadero, en el marco de la Norma Técnica Sectorial De Turismo -Destinos de Playa -Requisitos de Sostenibilidad (2014-2015).

La estructura teórica que direcciona la investigación es la sostenibilidad global aplicada al turismo, en la que se acudieron a los lineamientos dados en el Programa 21, los Criterios Globales de Turismo Sostenible, la Carta Mundial de Turismo Sostenible +20 de la OMT y los Principios de la Gobernabilidad Urbana establecidos por la ONU. Este último referente fue complementado con otros autores para llegar a la estructura esencial y reglas básicas que debe seguir un destino turístico para ser sostenible.

\section{Marco de referencia}

A continuación, se muestran algunos referentes teóricos que soportan los conceptos teóricos de desarrollo sostenible en el turismo y principios de gobernabilidad urbana desde la sostenibilidad.

\section{Breve inventario del concepto de desarrollo sostenible en el turismo}

Desde 1946 hasta el 2015, en períodos anuales, se han celebrado congresos, asambleas y reuniones de orden mundial, en los que se han establecido los lineamientos de políticas públicas de turismo sostenible, de los cuales se resaltan el Programa 21, los Criterios Globales de Turismo Sostenible y la Carta Mundial de Turismo Sostenible +20 .

El capítulo 17 de la sección II del Programa 21, "Protección de los recursos oceánicos", hace hincapié en la importancia de los océanos, sus amenazas y políticas de solución. El parágrafo de Zonas Costeras esboza las problemáticas en relación al ambiente dadas en el área:

(...) la pesca, el comercio, el transporte marítimo y el turismo crean a menudo problemas que amenazan el desarrollo sostenible: la contaminación química y bacteriana, los sedimentos de las tierras desbrozadas, la destrucción de los hábitat, la filtración de nutrientes que estimulan el exceso de crecimiento de plantas acuáticas y los asentamientos humanos pobres, hacinados y poco sanitarios. (ONU, 1992, p. 32)

Por su parte, los Criterios Globales de Turismo Sostenible constan de 34 criterios agrupados en cuatro políticas generales, "Demostrar una gestión sostenible eficaz; maximizar los beneficios sociales y económicos a la comunidad local y minimizar los impactos negativos; minimizar los impactos negativos al patrimonio cultural y maximizar los beneficios; y minimizar los impactos ambientales negativos y maximizar los beneficios" (Global Sustainable Tourism Council [GSTC], 2008, pp. 3-6).

Asimismo, la Carta Mundial de Turismo Sostenible +20 establece 13 acuerdos, de los cuales se resaltan algunas de sus propuestas:

El turismo es un motor de la economía mundial y especialmente de los países en desarrollo, por medio del cual se puede contribuir a la lucha contra la pobreza, mediante la utilización de los recursos y servicios locales; se protege la naturaleza y medio ambiente a través de la administración eficiente de los residuos sólidos y adopción de tecnología y modos de gestión innovadores para el uso del agua y la energía. Asimismo, se insta a la construcción de un turismo inteligente a través de las oportunidades que brindan la TIC. También es una fuerza motriz del patrimonio cultural, las artes y las industrias culturales y creativas (Cumbre Mundial de Turismo Sostenible, 2015, p.5)

En el mismo documento se enuncian los roles que deben cumplir los gobiernos y las organizaciones internacionales, los destinos y las comunidades locales, la industria del turismo, los consumidores, los investigadores, desarrolladores y formadores, las redes y las ONG. Las aplicaciones de los criterios de 
sostenibilidad también hacen parte de las cert ificaciones de calidad de playa. En el caso colombiano, la Norma Técnica Sectorial de Turismo (NTS-TS-001-2), base del presente trabajo, comprende 21 indicadores en las dimensiones ambiental, sociocultural, económica y de seguridad (figura 1).

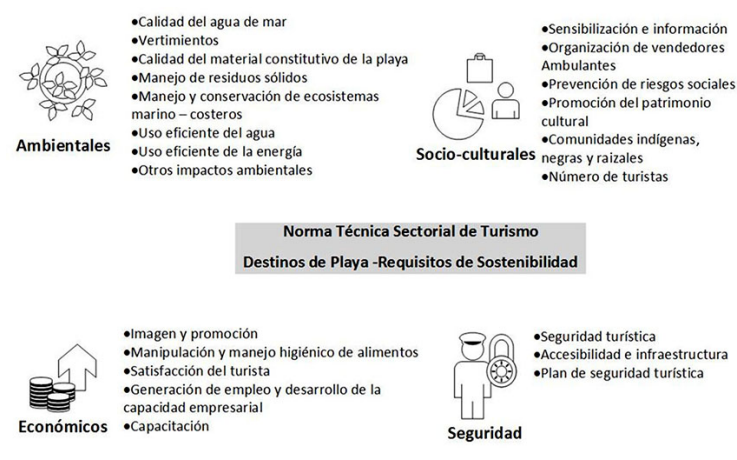

FIGURA 1.

Indicadores de la Norma Técnica Sectorial de Turismo (NTS-TS-001-2).

Fuente: elaboración propia a partir del Ministerio de Comercio, Industria y Turismo (Mincit) (2011).

El avance expuesto, a nivel de políticas de turismo sostenible, está sustentado principalmente en el hito histórico, que vale la pena resaltar por el impacto que ha tenido en las políticas de sostenibilidad. Se trata del reporte Nuestro Futuro Común de la ONU (1987), conocido como Reporte Brundtland, en el cual se definió el concepto de desarrollo sostenible, acogido también por el Banco Mundial y el Global Reporting Initiative. En Colombia, este concepto es definido por la Ley 99 de 1993, Artículo 3, o Ley ambiental colombiana, como

(...) el que conduzca al crecimiento económico, a la elevación de la calidad de la vida y al bienestar social, sin agotar la base de recursos naturales renovables en que se sustenta, ni deteriorar el medio ambiente o el derecho de las generaciones futuras a utilizarlo para la satisfacción de sus propias necesidades. (Ley 99, 1993, art. 3)

La base sobre la cual se construye la definición, y que debe ser el hilo conductor del crecimiento económico y de la calidad de vida de las personas, es el cuidado que se le debe prestar a la utilización de los recursos naturales y el medio ambiente, no solo pensando en el día de hoy sino en el futuro de nuestros sucesores. Desafortunadamente, esto dista del comportamiento de las empresas en la actualidad, donde el crecimiento económico se basa en la obtención de utilidades a base de la inadecuada utilización de los recursos naturales y explotación de las personas, lo que afecta su calidad de vida y dificulta la posibilidad de asegurar un futuro a sus descendientes. Este enfoque, trasladado al sector turismo, implica, entre otras actividades, prestar atención a los recursos naturales encaminados a la conservación de los ecosistemas marino-costeros, una revisión de la capacidad de carga, un uso eficiente de los servicios públicos con aprovechamiento de fuentes limpias provenientes de la energía solar, eólica e hídrica (mar y ríos); y vertimientos y manejo adecuado de los residuos sólidos. Por esta razón, "el desarrollo sostenible implica cambios, principalmente se necesita saber qué es lo que necesita sostenerse para cambiarlo y llegar al desarrollo que se necesita" (Gallopín, 2003, p.22). No basta con conocer las dimensiones de la sostenibilidad y sus indicadores; estos deben ser revisados en forma constante para que generen cambios en las actitudes, comportamientos y acciones en favor del bienestar común de los actores para garantizar el mantenimiento de los recursos naturales y aprovechamiento del patrimonio intangible.

\section{Principios de gobernabilidad urbana desde la sostenibilidad}

Desde hace más de dos décadas, el desarrollo urbano es analizado y ejecutado con lineamientos gerenciales de planificación y comercialización, lo que ha repercutido en el posicionamiento de las ciudades. Al respecto, Viken y Nyseth (2009) señalan que desde la década de 1980, la comercialización de la ciudad se ha convertido 
en un concepto clave en la planificación del desarrollo urbano y la gestión urbana, y Grogan y Proscio (2000) (en Rich \& Stoker, 2014) comentan que "en la práctica, la comercialización de la ciudad ha llegado a denotar las políticas de planificación que conduzcan al atractivo de la ciudad y la competitividad” (p.8). Esta visión gerencial debe ser complementada con el principio de oportunidad y el sentido de la urgencia, acciones necesarias para los territorios que desean adaptarse y transformarse a las necesidades del entorno con criterios de sostenibilidad. En este sentido, Perry y Eweje (2011) afirman que "para la creación de organizaciones sostenibles, las cuales deben ser caórdicas, término acuñado por (Hock, 1999) fundador de la VISA, donde la velocidad y la complejidad del cambio, es una mezcla constante de orden y el caos" (p.10).

Conrad y Thompson (2013) complementan la sugerencia al argumentar que el cambio empieza en la organización, donde la responsabilidad social debe ser la principal actividad, con criterios de transparencia, enfoque en los resultados en los ámbitos ambientales y sociales. Del mismo modo, recomiendan mantener el diálogo constante con las diferentes comunidades, comunicar sus causas e intenciones más que sus resultados e inspirar y capacitar en consumo de productos y servicios sostenibles.

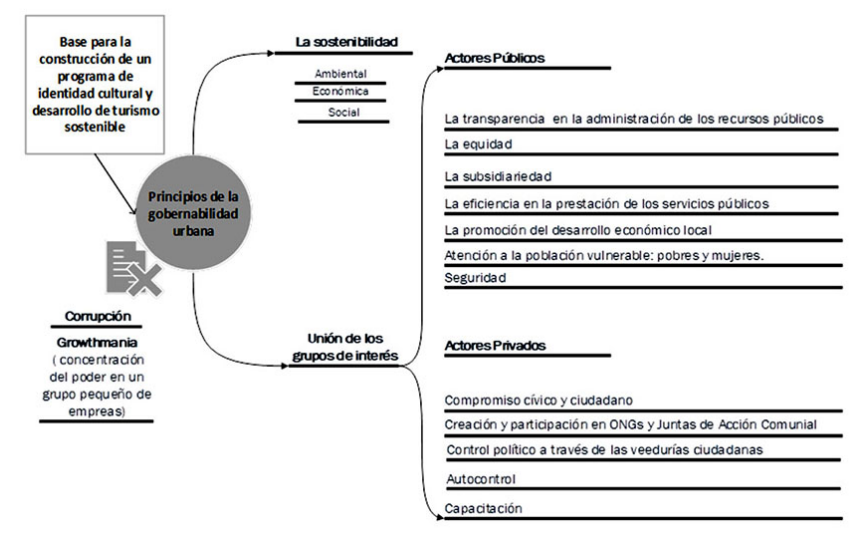

FIGURA 2.

Principios de la gobernabilidad urbana.

Fuente: elaboración propia a partir de los aportes teóricos de la ONU (2005)

Para ello es indispensable el trabajo articulado del talento humano que lo integra, mediante la generación del sentido de pertenencia por la ciudad, la construcción de alianzas público-privadas y el control a nivel individual y de las autoridades gubernamentales. Desde este contexto, Rich y Stoker (2014) señalan "como factores claves del mejoramiento de ciudades la autoayuda, el control local, desarrollo de la comunidad y de asociación pública-privada, las cuales son variables de éxito en ciudades" (p.37). A su vez, la estrategia planteada identifica las problemáticas sociales y motiva a las entidades con competencias complementarias, como la academia, la empresa y el gobierno; y a las de competencias similares que, por su experiencia, tienen un mayor avance en el sector, como las agencias de turismo, las agencias de viajes, los hoteles, a integrarse en función de las dimensiones de la sostenibilidad global. En virtud de lo anterior, O'Sullivan, Brady, Ray, Sikora y Murphy (2014) anotan que "las políticas tienen un amplio margen para la representación de la región de la ciudad, (...) con la coordinación horizontal y vertical de numerosas agentes públicos y privados institucionales, así como la integración espacial en las dimensiones sociales, ambientales y económicos" (p.307).

La unión de los actores genera sinergias que apuntan hacia un mismo norte, en beneficio de un objetivo en común, como es la de trabajar por la calidad de su entorno por medio de estrategias construidas por ellos mismos. En línea con lo anterior, Seixas y Albert (2013) advierten que

Es la capacidad y el compromiso de los actores públicos y privados, su inteligencia para acordar estrategias comunes para la construcción de proyectos movilizadores, su capacidad para identificar las fortalezas basadas en un propósito común, (...) lo que garantiza la calidad de las poblaciones locales. (p.6) 
Su estructuración se puede dar a partir de las organizaciones no gubernamentales y las juntas de acción comunal, mediante las cuales se pueden concretar sus propuestas.

En síntesis, el empoderamiento de los actores, su integración y la participación en ONGs, crean nuevas identidades socioculturales, desarrollan el liderazgo ejercido en su comunidad y elevan su rol de ciudadano al del político transparente, con sentido de comunidad y civismo por el territorio al que pertenecen. Según Cornwall (2002); Fung y Wright (2003); Houtzager et al. (2003); y Kabeer (2002), todos citados en Wit y Baud (2008), "Se están desarrollando nuevas identidades socioculturales en el proceso (...) donde el incremento de las Organizaciones de la Sociedad Civil (OSC) están contando con la participación de los ciudadanos con más fuerza que antes" (p.146).

Los argumentos anteriores exponen la estructura principal en la que descansa la gobernabilidad urbana, según lo enunciado por la Organización de las Naciones Unidas (2005), la cual se basa en la interdependencia y refuerzo mutuo de nueve principios, a saber:

(a) la sostenibilidad en todas las dimensiones del desarrollo urbano; (b) la subsidiariedad de la autoridad y los recursos al nivel apropiado más cercano; (c) la equidad de acceso a los procesos de toma de decisiones y las necesidades básicas de la vida urbana; (d) la eficiencia en la prestación de los servicios públicos y en la promoción del desarrollo económico local; (e) la transparencia y la rendición de cuentas de los responsables políticos y todas las partes interesadas; (f) el compromiso cívico y la ciudadanía en especial de las mujeres y los pobres; y (g) la seguridad de las personas y su ambiente. (p.58)

Este enfoque no solo evidencia la participación integral de los actores, sino que, además, expone otros factores que contribuyen al desarrollo urbano, como los servicios públicos, el énfasis en la atención a las mujeres y los pobres, y la seguridad. Así, una prestación eficiente de los servicios públicos, un menor número de personas en la categoría de pobres y una percepción alta de seguridad en un territorio denotan transparencia en el manejo de los recursos públicos por parte de los administradores locales. Un escenario opuesto a este, advertiría la posibilidad de focos de corrupción. Estos elementos, y en especial la corrupción, hacen parte de la medición de la competitividad de un país o una región. En este sentido, se puede afirmar que un territorio es más competitivo en la medida que su índice de corrupción es bajo.

Otro fallo del mercado o externalidad negativa es "la concentración del poder en un número relativamente pequeño de corporaciones, (...) orientadas por una economía de mercado, sin incorporación integral de los costos ambientales, denominada growthmania o la growthfetish" (Speth y Haas, 2006, p.48). Pese a estos agentes perturbadores, en contravía de un adecuado bienestar comunitario, los actores sociales pueden contribuir a su disminución o radicación, mediante la aplicación de los principios de la buena gobernabilidad expuestos anteriormente. Esta capacidad de la comunidad de resistir los impactos externos a su infraestructura social es denominada por Adger (2000), como se citó en Pike, Dawley y Tomaney (2010), como resiliencia social.

Finalmente, se plantea una estrategia adicional, y es cuestionarse en qué son fuertes las comunidades y sus entornos geográficos, cuáles son sus ejes misionales y, en ese orden de ideas, proponer un sistema de identidad que contribuya al desarrollo del turismo. Clancy (2009), en el libro Brand New Ireland?, establece que "en un período de cambios rápidos como consecuencia de la globalización, se explora el papel del turismo y se utiliza como una lente para observar la formación de la identidad nacional” (p.1). Además, y pese a que la globalización impone pautas y gustos iguales, el desarrollo de los territorios es particular y se ve impactado por los valores culturales de los individuos, quienes prefieren permanecer la mayor parte de su vida en su área de origen. Así, Boisier (citado en Calvento \& Ochoteco, 2009) señala tres características que hacen del territorio un instrumento que la globalización no ha vuelto anacrónico:

las personas atraviesan la mayoría de las etapas de su vida en un territorio concreto de no más de $500 \mathrm{~km}$ de radio, la localización de las empresas ha llevado a valorar las condiciones de los territorios en los cuales se asientan y la última característica se acerca a variables culturales e identitarias. (pp.61-62) 
Este comportamiento territorial de los seres humanos, de establecerse en un entorno geográfico limitado y crear sus propias redes económicas, genera hábitos y comportamientos similares que se traducen en una impronta de identidad cultural, la cual no es solo un atributo que se debe propender por su conservación, sino que, además, puede ser objeto de usufructo en beneficio de las generaciones actuales y futuras.

\section{Metodología}

En este artículo se muestran las debilidades, a partir de la revisión de cada uno de los indicadores de la Norma Técnica Sectorial de Turismo (NTS-TS-001-2), en las dimensiones ambiental, sociocultural, económica y de seguridad. Los datos provienen de fuentes primarias, como la observación directa y algunos resultados de las encuestas aplicadas a turistas, empresarios y residentes; así como de informes de investigaciones y boletines técnicos. En forma complementaria, se analizan los resultados de la percepción de imagen del Rodadero, en el que se tuvieron en cuenta 16 variables, mencionadas a continuación: en la dimensión ambiental, aseo, uso de espacio público, nivel de contaminación auditiva, nivel de contaminación visual, y nivel de contaminación ambiental; en la dimensión económica, calidad en alojamiento, calidad en alimentos y bebidas, calidad de los atractivos turísticos, servicio de guías, actitud y amabilidad del residente frente al turista; en la dimensión sociocultural, puntos de información y señalización, cuidado del patrimonio cultural, natural e histórico; y finalmente en dimensión seguridad, seguridad, accesibilidad al destino, vías de transporte y movilidad, servicios médicos y centro de salud y facilidades bancarias. Para ello, se utilizó la técnica de carácter correlacional, denominada Análisis Factorial de Correspondencias Múltiples, mediante el software SPAD WIN 3.1.

\section{Resultados}

A continuación se muestra la fase diagnóstica con respecto a las debilidades con base en la NTS-TS-001-2, y la percepción de imagen de los turistas, empresarios y residentes en las dimensiones ambiental, sociocultural, económica y de seguridad.

\section{Dimensión Ambiental}

A la luz de la NTS-TS-001-2 de turismo se revisaron los ítems calidad de agua de mar, vertimientos, calidad del material constitutivo de la playa, manejo de residuos sólidos, manejo y conservación de ecosistemas marino - costeros, uso eficiente del agua, uso eficiente de la energía y otros impactos ambientales, con los siguientes hallazgos. Según Arregocés (2009), existe una dificultad en la recuperación de los pastos marinos mixtos por arrastre de contaminantes provenientes de sistemas hídricos; una afectación del ecosistema por alta presión antrópica, con aumento y mala disposición de residuos orgánicos e inorgánicos, y por el desarrollo de actividades pesqueras. Además, se observa deficiencia en el abastecimiento de los servicios públicos por aumento en la capacidad de carga, especialmente en temporada alta. La contaminación auditiva, visual y ambiental es una debilidad (encuestas aplicadas a los turistas, empresarios y residentes), los turistas desconoces los planes de comparendos ambientales y su deber con el cuidado ambiental; y hay poca divulgación de las normas, sanciones y campañas ambientales. Importar tabla

De forma complementaria, se evaluó la percepción de turistas, empresarios y residentes, en relación al aseo, uso del espacio público, nivel de contaminación auditiva, nivel de contaminación visual, y nivel de contaminación ambiental. En los resultados obtenidos se denota un efecto significativo con valores absolutos mayores a 1,96 ee, así como de los diferentes tipos de actores, salvo los empresarios, quienes son los únicos que 
no influyen significativamente en dicho componente. De lo anterior se resalta que las variables seleccionadas dentro del componente ambiental generan un efecto de percepción significativa en los diferentes actores y se deben trabajar en forma integral, con el fin de propender por una mejora de la dimensión en mención. En el dendograma de la dimensión ambiental (figura 3 y tabla 1) se observa la consolidación de cinco tipologías de grupos o calificaciones, en función de las variables:

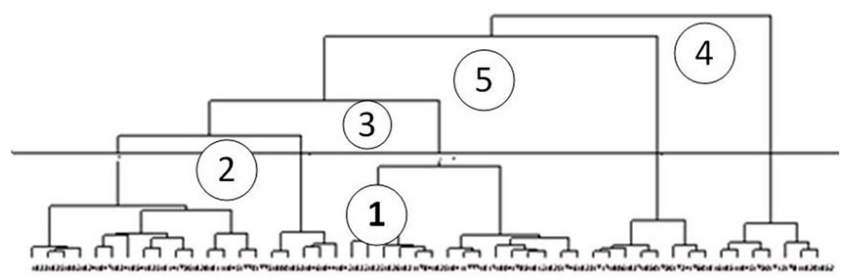

FIGURA 3.

Dendograma de la dimensión ambiental.

Fuente: elaboración propia

TABLA 1.

Calificación de la dimensión ambiental

\begin{tabular}{cccc}
\hline $\mathbf{1}$ & Regular & 37,61 & \\
\hline $\mathbf{2}$ & Buena & 23,11 & $\begin{array}{c}\text { Turistas } \\
\text { junio }\end{array}$ \\
\hline $\mathbf{3}$ & Mala & 19,75 & Residentes \\
\hline $\mathbf{4}$ & Pésima & 12,28 & Residentes \\
\hline $\mathbf{5}$ & Excelente & 7,25 & $\begin{array}{c}\text { Turistas } \\
\text { diciembre }\end{array}$ \\
\hline & Total & 100 & \\
\hline
\end{tabular}

Fuente: elaboración propia

Finalmente, la mayor calificación en la dimensión ambiental es regular (37,61\%), lo que requiere medidas de mejoramiento, algunas de ellas propuestas en el presente análisis.

\section{Dimensión Sociocultural}

La NTS-TS-001-2 determina seis indicadores a saber: sensibilización e información, organización de vendedores ambulantes, prevención de riesgos sociales, promoción del patrimonio cultural; comunidades indígenas, negras y raizales, y número de turistas. En este contexto, se definió el siguiente diagnóstico. Según las encuestas aplicadas a los turistas, empresarios y residentes son las debilidades del sector, la mínima señalización e información de sitios turísticos, la falta de servicios médicos y centros de salud, y el poco cuidado del patrimonio cultural e histórico. De igual manera, figura la invasión del espacio púbico por los vendedores ambulantes, que en temporada alta pueden estar en un promedio de 2000 , el cual es un problema social con alto impacto en la imagen del destino turístico. También están el poco interés de las autoridades competentes para la organización de actividades recreativas y culturales en las diferentes épocas del año y los pocos procesos de consulta previa a las entidades étnicas, en el desarrollo demográfico de destinos turístico de playa, que impliquen aspectos socioculturales, económicos y sociales.

Adicionalmente se analizaron la percepción de los turistas, empresarios y residentes de los ítems, puntos de información y señalización, y cuidado patrimonio cultural, natural e histórico, y se obtuvieron los siguientes hallazgos: se denota un efecto significativo con valores absolutos mayores a 1,96 ee en las variables propuestas; 
sin embargo, se presenta una apreciación positiva de los turistas de diciembre frente a la percepción negativa del resto de actores. En relación al dendograma de la dimensión sociocultural (figura 4 y tabla 2), se identifican tres tipos de calificaciones de los actores:

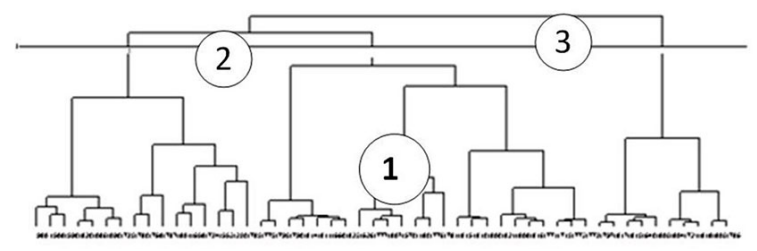

FIGURA 4.

Dendograma de la Dimensión Sociocultural.

Fuente: elaboración propia

TABLA 2.

Calificación de la Dimensión Sociocultural

\begin{tabular}{|c|c|c|c|}
\hline 1 & $\begin{array}{l}\text { Bueno } \\
\text { Regular }\end{array}$ & 50.43 & \\
\hline 2 & Mala & 38.35 & \\
\hline 3 & Excelente & 11.22 & $\begin{array}{l}\text { Turistas } \\
\text { diciembre }\end{array}$ \\
\hline
\end{tabular}

Fuente: elaboración propia

Como se puede ver en la tabla anterior, la mayor calificación es de bueno-regular, otorgada por el 50,43\% de los encuestados.

\section{Dimensión Económica}

En la dimensión económica, la NTS-TS-001-2 tiene en cuenta la imagen y promoción, la manipulación y el manejo higiénico de alimentos, la satisfacción del turista, la generación de empleo y el desarrollo de la capacidad empresarial (capacitación). En virtud de lo anterior, se tienen los siguientes hallazgos: según los empresarios, son debilidades del destino turístico la actitud y la amabilidad del residente ante el turista; los costos de alojamiento en los apartamentos, los cuales hacen que se generen situaciones de orden y sostenibilidad del destino; y el poco control por parte del gobierno local en relación con la manipulación y el manejo higiénico de alimentos. El turista está insatisfecho por la proliferación de los vendedores ambulantes y la falta de cultura ciudadana; los empleos son precarios, mal remunerados, estacionales y no cumplen con las normas legales; la calidad en los servicios turísticos es baja, y el posicionamiento de Santa Marta como destino turístico; débil (Herrera, Leguizamón, \& Duque, 2010); la participación de la población en edad de trabajar es baja (Maldonado, 2012), el nivel educativo más representativo entre turistas y oferentes del servicio es la secundaria y, por último, los programas de formación y capacitación hacia los actores y prestadores de servicios, en cuanto a la calidad y sostenibilidad de las playas, tienen un nivel medio de continuidad y pertinencia.

Adicional a lo anterior, se analizó la percepción de turistas, empresarios y residentes en relación a la calidad en alojamiento, calidad en alimentos y bebidas, calidad de los atractivos turísticos, servicio de guías, y actitud y amabilidad del residente frente al turista. A partir del resultado de dicho análisis, se denota un efecto significativo con valores absolutos mayores a 1,96 ee, así como de los diferentes tipos de actores, los cuales influyen significativamente sobre los niveles de respuesta con respecto al componente económico, 
salvo los empresarios, quienes no generan una percepción significativa, posiblemente por su capacidad de adaptación a las condiciones con respecto a las variables bajo estudio. No obstante, existe una tendencia general a valorar dichas variables en niveles de regular a bueno, en la cual se presenta una contraposición de percepción de residentes, con una percepción desfavorable con respecto a los turistas de las temporadas de junio y diciembre, quienes, en términos generales, perciben dichas variables económicas de manera positiva. Al realizar el dendograma de la dimensión económica (figura 5 y tabla 3), se identifican cuatro tipologías:

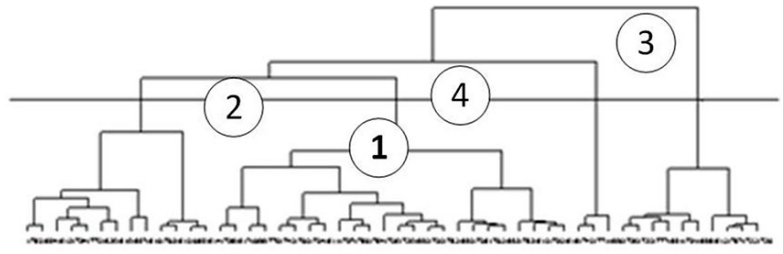

FIGURA 5

Dendograma de la dimensión económica.

Fuente: elaboración propia

TABLA 3.

Calificación de la dimensión económica del Rodadero

\begin{tabular}{lllc}
\hline 1 & Buena & 41,5 & $\begin{array}{c}\text { Empresarios } \\
\text { y turistas de junio }\end{array}$ \\
\hline 2 & Excelente & 29,3 & Turistas \\
\hline 3 & Regular & 26,74 & Residentes \\
\hline 4 & Mala & 2,46 & Residentes \\
\hline & Total & $\mathbf{1 0 0}$ & \\
\hline
\end{tabular}

Fuente: elaboración propia

Según la tabla 3, la mayor calificación es buena, dada por el 41,5\% de los encuestados.

\section{Dimensión Seguridad}

A continuación, se presenta el diagnóstico a la luz de los ítems seguridad turística, accesibilidad e infraestructura, y plan de seguridad turística de la NTS-TS-001-2: la policía de turismo no cuenta con personal bilingüe en su punto de información, ni en las jornadas que hace para la prevención de robos; la venta informal genera inseguridad a los turistas, existe una deficiencia en la infraestructura para la oferta turística, y la gestión institucional es débil (Herrera, Leguizamón, \& Duque, 2010); el destino de playa no cuenta con zonas especiales para discapacitados, y hay poco personal de salvamento, planes de emergencia y baños.

Posteriormente, se añadió el análisis de percepción de turistas, empresarios y residentes de las variables seguridad, accesibilidad al destino, vías de transporte y movilidad, servicios médicos y centros de salud, y facilidades bancarias. Este genera un efecto significativo con valores absolutos mayores a 1,96. En el plano factorial (figura 6 y tabla 4), se muestra un efecto de contraste en la percepción, donde la calificación de bueno a excelente tiende a presentarse en los turistas, mientras que los residentes perciben un efecto negativo en las condiciones de seguridad. Las calificaciones se consolidan en tres tipologías de actores, como se observa en el dendograma de la dimensión seguridad (figura 6). 


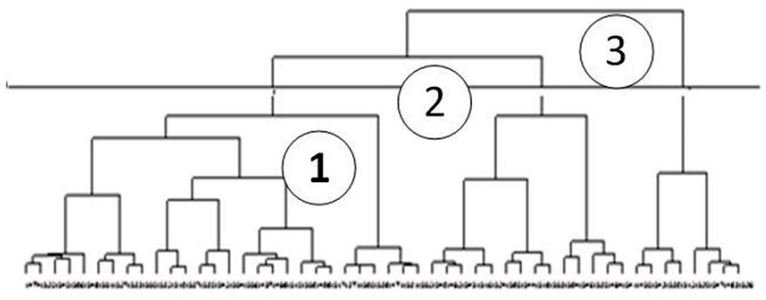

FIGURA 6.

Dendograma de la dimensión seguridad.

Fuente: elaboración propia

TABLA 4.

Calificación de la dimensión seguridad del Rodadero

\begin{tabular}{|c|c|c|c|}
\hline Tipología & Calificación & $\mathbf{\%}$ & \begin{tabular}{c} 
Clase de actor \\
\hline 1
\end{tabular} \\
\hline 2 & Buena y Regular & 63,89 & $\begin{array}{c}\text { Empresarios y } \\
\text { turistas de junio }\end{array}$ \\
\hline 3 & Excelente & 19,55 & $\begin{array}{c}\text { Turistas } \\
\text { diciembre }\end{array}$ \\
\hline & Mala & 16,56 & Residentes \\
\hline
\end{tabular}

Fuente: elaboración propia

La mayor calificación es de bueno-regular, otorgada por el 63,89\% de los encuestados.

\section{Discusión}

Se logró realizar un análisis del balneario El Rodadero, con respecto a la NTS-TS-001-2, lo que evidenció que las dimensiones de mejor percepción por parte los empresarios, turistas y residentes son la económica y la de seguridad, frente a las de menor percepción, la ambiental y la sociocultural (tabla 5).

TABLA 5.

Percepción de la imagen del Rodadero (cifras en \%)

Buena y

Excelente regular

Mala y pésima

\begin{tabular}{lrrr}
\hline Ambiental & 7,25 & 60,72 & 32,03 \\
\hline Sociocultural & 11,22 & 50,43 & 38,35 \\
\hline Económica & 29,30 & 68,24 & 2,46 \\
\hline Seguridad & 19,55 & 63,89 & 16,56 \\
\hline
\end{tabular}

Fuente: elaboración propia

Para lo obtención de los resultados, se acudió a la técnica denominada Análisis Factorial de Correspondencias Múltiples, utilizada en el trabajo de Andrade et al. (2015), titulado "Características del suelo en los cultivos de cafés especiales del municipio de Calarcá-Colombia”. 
El diagnóstico realizado es un esbozo descriptivo de los aspectos negativos de la Norma Técnica Sectorial de Turismo (NTS-TS-001-2), en el cada una de las variables puede ser objeto de trabajos especializados a nivel científico. De hecho, el mismo análisis se alimenta de los trabajos de Arregocés (2009), en relación a la dimensión ambiental; y de Maldonado (2012), en relación con los vendedores ambulantes en la dimensión sociocultural. Asimismo, puede ser profundizado con otras miradas científicas, como lo realizado en el trabajo titulado "Selección y propuesta de parámetros para la determinación de la calidad ambiental en playas turísticas del Caribe Colombiano”, por Hurtado-García, Botero y Herrera (2009), en el que analizaron la NTS y la playa de El Rodadero. Los autores revisaron instrumentos normativos de calidad de playas turísticas en diferentes países, y criterios de selección y análisis de hábitos de visitantes en dos playas de referencia, y obtuvieron un listado de parámetros físico-químicos y microbiológicos que integra la zona emergida dentro de la medición de calidad de playas turísticas, con lo cual se contribuye a la adopción de futuras reglamentaciones relativas a la actividad turística en las playas del Caribe colombiano. Igualmente, los resultados pueden ser complementarios a los obtenidos en el trabajo "Metodología de cálculo de la capacidad de carga turística como herramienta para la gestión ambiental y su aplicación en cinco playas del Caribe Norte Colombiano" de Botero-Saltarén, Hurtado-García, González-Porto, Ojeda-Manjarrés y Díaz-Rocca (2008), en el que determinan la capacidad de carga del Rodadero de 3,032 visitantes frente a una capacidad de 814 personas, la deficiencia en el servicio de alcantarillado y tratamiento de aguas residuales; y los bajos niveles de calidad microbiológica en agua y arena, que generan contaminación del agua de mar. En este mismo sentido, en "Los esquemas de certificación de playas turísticas en América Latina: Evaluación del componente sociocultural y el nivel participativo", a cargo de Zielinski y Díaz (2014), las autoras emiten el siguiente juicio de la NTS-TS-001-2:

Resulta una norma con altas exigencias, conocimientos especializados y una significativa inversión. Técnicamente es muy completa y de lograrse implementar permitiría unos niveles óptimos de gestión en la playa. Nombra a la comunidad en un plan de capacitación que garantice el buen desempeño del sistema, pero no va más allá. Se considera que es una norma que contiene estándares del deber ser más que del hacer en lo cual se incluye el fortalecimiento de las comunidades costeras en la gestión adecuada de las playas. (p.170)

Se destaca, también, el aporte de Gutiérrez-Fernández y Sánchez (2016), quienes observan la playa como una organización en los niveles de gestión estratégico, táctico y operacional, y describen cada uno de los programas, y sus responsables, en la implementación de la NTS-TS-002 de la Playa La Aguada, ubicada en el Parque Nacional Natural de Utría, en el Departamento del Chocó - Colombia, cuyo principal atractivo turístico es el avistamiento de ballenas jorobadas. Es la primera playa certificada en Colombia en el esquema de NTS-TS-002. El modelo de implementación debe ser adoptado por El Rodadero y otras playas, con miras a mejorar sus niveles de calidad y competitividad.

Estos análisis contribuyen a la aplicación del Decreto 376 del 4 de diciembre de 2015, de la ciudad de Santa Marta, cuyo contenido (al menos, parte de él) se basa en la NTS-TS-001-2; y a la afiliación de la ciudad al Programa Ciudades Emergentes y Sostenibles del Banco Interamericano de Desarrollo (BID), en el mismo año.

\section{Conclusiones}

El balneario El Rodadero no es ajeno a las problemáticas enunciadas en la Protección de los recursos oceánicos, del Programa 21. Así, la falta de conciencia ciudadana por parte de turistas, residentes y vendedores ambulantes amenazan la sostenibilidad ambiental. Esta amenaza se evidencia en la disminución del área de playa por erosión y escorrentía producida por el incremento y la mala disposición de los residuos orgánicos e inorgánicos, la elevada presión antrópica en temporada alta y la dificultad en la recuperación de los parches de pastos marinos mixtos (Arregocés, 2009). 
Del mismo modo, se muestra una gestión sostenible débil en relación a los Criterios Globales de Turismo Sostenible. En ese sentido, se evidencia la falta de aplicación de las medidas de control por parte del Estado, en función del cumplimiento de los requisitos legales con respecto a la informalidad en tres niveles: ventas, guías turísticos y alojamiento. En ese orden de ideas, se observa una proliferación de las ventas ambulantes, un ofrecimiento de servicios turísticos por personas que no cuentan con la autorización legal y una prestación del servicio de alojamiento por parte de personas naturales propietarias de apartamentos que nos están inscritos en Cámara de Comercio. La informalidad descrita afecta los entes económicos formales, los cuales aportan al desarrollo económico mediante la generación de empleos, aporte de impuestos y mejoramiento de la imagen del destino turístico. Con respecto al patrimonio cultural, la zona Caribe, en general, es rica en intangibles expresados en la gastronomía, danzas y música. Sin embargo, este baluarte es poco aprovechado por la comunidad samaria, pues se limitan a la elaboración de artesanías, producción de shows de manera informal y ofrecimiento de algunos productos como parte de la oferta gastronómica en algunos hoteles y restaurantes.

De acuerdo a los lineamientos dados en la Carta Mundial de Turismo Sostenible +20 , se debe luchar por la erradicación de la pobreza, que en este caso está representada, especialmente, por los vendedores ambulantes. En la medida que se les brinden oportunidades de formalización a partir de la generación de empleos dependientes o independientes ofertados en la misma zona, se contribuirá al mejoramiento de sus ingresos y, por ende, al incremento de su calidad de vida. Así mismo, se debe proteger la naturaleza y el medio ambiente con investigación y con una promoción de una cultura ciudadana enfocada en la reutilización y reciclaje de los residuos sólidos. Se debe, también, aprovechar la tecnología en dos ámbitos: el primero, en función del desarrollo y aprovechamiento de energías limpias y sostenibles, teniendo en cuenta que la zona es rica en energía eólica e hídrica (mar); y el segundo, relacionado con el aprovechamiento de las Tecnologías de la Información (TIC) para desarrollar un turismo inteligente, en el cual se implementen aplicaciones, redes sociales, shows en tercera dimensión, entre otros.

Adicional a lo anterior, existen otros factores adversos que afectan los principios de la gobernabilidad urbana (ONU, 2005) del destino turístico. El ranking nacional de competitividad ubica a el Magdalena, donde se encuentra El Rodadero, en el puesto 21/25 de los departamentos analizados, e identifica entre sus debilidades la calidad de la educación pública y la producción de patentes y diseños industriales (Consejo Privado de Competitividad [CPC], 2015). En el orden internacional, Schwab (2014), en The Global Competitiveness Report 2014-15 del World Economic Forum, ubica a Colombia en la posición 66 entre 144 países evaluados, y señala los factores problemáticos calificados con mayor representación para el desarrollo de los negocios: la corrupción (19,3\%), el suministro inadecuado de infraestructura (4,3\%) y la ineficiencia en la burocracia gubernamental (12,2\%). Regresando al caso del Magdalena, con respecto al tamaño de las empresas, según la Cámara de Comercio de Santa Marta para el Magdalena (2015), en el 2014, en un universo de 26\#39, el 96,8\% son microempresas; el 3\%, pymes; y el 0,2\%, grandes empresas. En estos dos últimos grupos, se encuentran clasificadas las 200 empresas que más generan desarrollo en el Magdalena, con una participación en ventas y utilidades del 93,4\%, lo que denota "growthmania", o concentración del poder en un número relativamente pequeño de corporaciones. Pese a las circunstancias negativas, el balneario El Rodadero ha demostrado capacidad de resiliencia social a través del tiempo. Muestra de ello es que es un ícono turístico a nivel nacional y un atractivo para la inversión nacional y/o extranjera. Sin embargo, debe reinventar su direccionamiento con el fin de adaptarse a los nuevos cambios de la globalización, pues, como lo manifiesta Clancy (2009), el turismo y el desarrollo de la identidad cultural se convierten en una estrategia para hacer frente a los cambios globales.

Ahora bien, si El Rodadero pretende mejorar su posicionamiento en el mercado de acuerdo a sus ventajas comparativas en beneficio del turismo, se debe planificar y comercializar siguiendo lineamientos gerenciales basados en la sostenibilidad, que lo conduzcan a la consolidación de un sistema de identidad, mejoramiento de su imagen y competitividad a partir del diálogo, la unión y el cambio en las actitudes y aptitudes de cada uno 
de los actores. En este cambio deben primar la responsabilidad social con criterios de transparencia y enfoque en los resultados, la generación del sentido de pertenencia por la ciudad, la construcción de alianzas públicoprivadas y el control a nivel individual y de las autoridades gubernamentales. El empoderamiento de los actores y las asociaciones verticales en turismo y asociaciones horizontales con competencias complementarias en medio ambiente, seguridad, salud, educación, deporte y política crearán nuevas sinergias con sentido de comunidad y liderazgo, pero, para lograr esto, se deben aprovechar las redes económicas existentes, en beneficio de las generaciones actuales y futuras.

\section{Referencias}

Alcaldía de Santa Marta. (4 de diciembre de 2015). Decreto 376 de Ordenamiento de Playas en el Distrito de Santa Marta.

Andrade, A. L., Cerón-Chacón, V., Gómez-Duque, J. R., Hormiga-García, D. N., Mejía-Giraldo, L. M., y TorresValenzuela, L. S. (2015). Características del suelo en los cultivos de cafés especiales del municipio de CalarcáColombia. UGCiencia, 21, 29-38. https://doi.org/10.18634/ugcj.21v.1i.421

Arregocés, L. (2009). Caracterización y diagnóstico ambiental del Balneario "El Rodadero", Bahia Gaira, Santa Marta, Caribe Colombiano (Tesis de pregrado). Universidad Jorge Tadeo Lozano, Santa Marta, Colombia.

Calvento, M. y Ochoteco, M. (2009). Una aproximación a la construcción de marca-ciudad como estrategia de inserción nacional e internacional. Economia, Sociedad y Territorio, 9(29), 59-87.

Cámara de Comercio de Santa Marta para El Magdalena (2015). Las 200 empresas más grandes del Magdalena. Recuperado de https://www.ccsm.org.co/servicios-empresariales/servicios-ofertas-empresariales/estudios-econ omicos/investigaciones-e-indicadores-economicos/send/78-investigaciones/48088-las-200-empresas-mas-gran des-del-magdalena.html

Clancy, M. (2009). Brand New Ireland? Tourism, Development and National Identity in the Irish Republic. Farnham, Surrey, England: Routledge.

Congreso de Colombia. (1993). Por la cual se crea el Ministerio de Medio Ambiente, se reordena el Sector Público encargado de la gestión y conservación del medio ambiente y los recursos naturales renovables, se organiza el Sistema Nacional Ambiental -SINA- y se dictan otras disposiciones. [Ley 99 de 1993]. Recuperado de http://w ww.humboldt.org.co/images/documentos/pdf/Normativo/1993-12-22-ley-99-crea-el-sina-y-mma.pdf

Conrad, C. y Thompson, M. (2013). The New Brand Spirit: How Communicating Sustainability Builds Brands, Reputations And Profits. Burlington: Gower.

Consejo Privado de Competitividad (CPC). (2015). Índice Departamental de Competitividad 2014. Recuperado de h ttps://compite.com.co/idc/

Cumbre Mundial de Turismo Sostenible. (2015) Carta Mundial de Turismo Sostenible. Recuperado de http://cartamundialdeturismosostenible2015.com/wp-content/uploads/2016/05/Carta-Mundial-de-Turi smo-Sostenible-20.pdf

Gallopín, G. (2003). Sostenibilidad y desarrollo sostenible: Un enfoque sistémico. Recuperado de https://repositorio.ce pal.org/bitstream/handle/11362/5763/S033120_es\%20.pdf?sequence=1\&isAllowed=y

Hurtado-García, Y. P., Botero, C. M., y Herrera, E. (2009). Selección y propuesta de parámetros para la determinación de la calidad ambiental en playas turísticas del Caribe Colombiano. Ciencia en su PC, (4), 42.53.

Global Sustainable Tourism Council (GSTC). (2008). Criterios Globales de Turismo Sostenible. Recuperado de https ://www.gstcouncil.org/los-criterios-globales/

Gutiérrez -Fernández, F. y Restrepo-Sánchez, N. (2016). Implementación de sistemas de gestión integral sustentable para destinos turísticos, caso de estudio Parque Nacional Natural Utría, playa La Aguada-Colombia. Revista Espiga, 15(31), 13-29 
Herrera, B., Leguizamón, M., y Duque, R. (2010). Estudio de la hotelería paralelo en el distrito turistico, cultural e histórico de santa Marta-zona de El Rodadero-propuesta de un modelo de intervención. Recuperado de https://fo ntur.com.co/aym_document/aym_estudios_fontur/ESTUDIO_PARA_HOTELERIA.PDF

Maldonado, A. (2012). Caracterización socioeconómica de los vendedores informales del sector turistico de El Rodadero. Santa Marta: Ministerio de Comercio Industria y Turismo y Universidad del Magdalena

Martínez, C. (2013). Diagnóstico actualizado y propuestas de manejo integrado para el Balneario "El Rodadero", Santa Marta, Caribe Colombiano (Tesis de pregrado). Universidad Jorge Tadeo Lozano, Santa Marta, Colombia.

Ministerio de Comercio, Industria y Turismo (Mincit) y Departamento Nacional de Planeación (DNP). (2014). Plan sectorial de turismo 2014-2018 "turismo para la construcción de la paz". Recuperado de http://www.mincit.gov.co/minturismo/loader.php?IServicio=Documentos\&lFuncion=verPdf\&id=71713 \&name=PLAN_SECTORIAL_DE_TURISMO_2014-2018_16_DE_SEPTIEMBRE_DE_2014.pdf\&prefij $\mathrm{o}=$ file

Ministerio de Comercio, Industria y Turismo (Mincit). (2011). Norma Técnica Sectorial Colombiana NTS-TS 001-2 Recuperado de http://www.minambiente.gov.co/images/normativa/Otros/NTC/2006/NTSTS_002_2006.p df

Organización de las Naciones Unidas (ONU). (1987). Desarrollo y Cooperación Económica Internacional: Medio Ambiente. New York: United Nations Publications.

Organización de las Naciones Unidas (ONU). (2005). Urban environmental governance for sustainable development in Asia and the Pacific: A regional overview. New York: United Nations Publications.

Organización de las Naciones Unidas (ONU). (1992). Programa 21. Recuperado de https://sustainabledevelopmen t.un.org/content/documents/1718a21_summary_spanish.pdf

O'Sullivan, B., Brady, W., Ray, K., Sikora, E., y Murphy, E. (2014). Scale, Governance, Urban Form and Landscape: Exploring the Scope for an Integrated Approach to Metropolitan Spatial Planning. Planning Practice and Research, 29(3), 302-316. https://doi.org/10.1080/02697459.2014.929846

Perry, M. y Eweje, G. (2011). Business and Sustainability: Concepts, Strategies and Changes. Bingley, UK: Emerald Group Publishing Limited.

Pike, A., Dawley, S., y Tomaney, J. (2010). Resilience, adaptation and adaptability. Cambridge Journal of Regions, Economy and Society, 3(1), 59-70. https://doi.org/10.1093/cjres/rsq001

Rich, M. y Stoker, R. (2014). Collaborative Governance For Urban Revitalization: Lessons From Empowerment Zones. Ithaca: Cornell University Press.

Botero-Saltarén, C. B., Hurtado-García, Y., González-Porto, J., Ojeda-Manjarrés, M., y Díaz-Rocca, L. H. (2008). Metodología de cálculo de la capacidad de carga turística como herramienta para la gestión ambiental y su aplicación en cinco playas del caribe norte Colombiano. Gestión y Ambiente, 11(3), 109-126.

Schwab, K. (2014). The Global Competitiviness Report 2014-15. Switzerland: World Economic Forum. Recuperado de http://www3.weforum.org/docs/WEF_GlobalCompetitivenessReport_2014-15.pdf

Seixas, J., y Albert, A. (2013). Urban Governance in Southern Europe. Burlington: Routledge.

Speth, J. y Haas, P. (2006). Global Environmental Governance: Foundations of Contemporary Environmental Studies. Washington: Island Press

Viken, A. y Nyseth, T. (2009). Place Reinvention: Northern Perspectives. Farnham, Inglaterra: Routledge.

Wit, J. y Baud, I. (2008). New Forms of Urban Governance in India: Shifts, Models, Networks and Contestations. New Delhi: SAGE Publications India Pvt., Ltd.

Zielinski, S. y Díaz, M. (2014). Los esquemas de certificación de playas turísticas en América Latina: Evaluación del componente socio-cultural y el nivel participativo. Estudios y perspectivas en turismo, 23(1), 156-175. 


\section{Notas}

* Artículo de investigación. Hace parte del proyecto Hacia la construcción de la marca Rodadero en el marco de la norma técnica sectorial de turismo ¿Destinos de playa requisitos de sostenibilidad?, financiado por la Dirección de Investigación, Extensión y Creatividad de la Universidad Jorge Tadeo lozano de Bogotá.

Licencia Creative Commons CC BY 4.0

Cómo citar este artículo: Sánchez, L. M. (2018). Sostenibilidad del balneario El Rodadero, Santa Marta, Colombia. Ambiente y Desarrollo, 22(42), 1-15. https://doi.org/10.11144/Javeriana.ayd22-42.sber 